\title{
Early onset recall pneumonitis during targeted therapy with sunitinib
}

\author{
Takeshi Yuasa1*, Shinichi Kitsukawa', Gen Sukegawa1', Shinya Yamamoto', Keita Kudo², Kazunari Miyazawa², \\ Takuyo Kozuka ${ }^{3}$, Sohei Harada ${ }^{4}$ and Junji Yonese ${ }^{1}$
}

\begin{abstract}
Background: Sunitinib interacts with radiation therapy, leading to synergism of the toxicities of these treatments. Radiation recall pneumonitis is a rare but serious complication of targeted therapy with tyrosine kinase inhibitors.

Case presentation: The case of a patient with metastatic renal cell cancer (RCC) who developed recall pneumonitis on the first cycle of systemic sunitinib treatment is reported here. A 65-year-old man with RCC and bone metastasis underwent radiation therapy on his thoracic vertebrae (Th5-8) with a total dose of $24 \mathrm{~Gy}$. Sunitinib (37.5 mg) was started 14 days after completing the radiation therapy. On the 14th day of sunitinib treatment, the patient developed progressive fever with worsening of dyspnea and general weakness. Treatment with pulse administration of prednisolone 1,000 mg for 3 days was initiated. Thereafter, the symptoms and the radiological findings regarding the interstitial filtration gradually improved over 7 days.

Conclusion: To our knowledge, this is the first report of early onset recall pneumonitis during sunitinib therapy. At present, how sunitinib interacts with radiation therapy remains unclear. The possibility that tyrosine kinase inhibitor therapy, including with sunitinib, after radiation therapy may lead to adverse effects should be kept in mind.
\end{abstract}

Keywords: Sunitinib, Renal cell cancer, Recall pneumonitis, Radiation, Radiation pneumonitis

\section{Background}

Renal cell cancer (RCC) is the most lethal of the urological malignancies, and its incidence is currently increasing [1]. Recently, several new targeted agents were introduced in the clinical treatment of metastatic RCC. One of these agents, sunitinib, is an orally administered tyrosine kinase inhibitor with multiple targets, including vascular endothelial growth factor receptor (VEGFR)-1, VEGFR-2, VEGFR-3, platelet-derived growth factor receptor (PDGFR)- $\alpha$, and PDGFR- $\beta$. Sunitinib is one of the most widely prescribed drugs for the treatment of metastatic RCC [2]. The adverse effects of sunitinib include fatigue, bone marrow suppression, hand-foot syndrome, stomatitis, hypertension, and hypothyroidism [3]. Interstitial lung disease is a rare but serious complication of targeted therapy with tyrosine kinase inhibitors, including sunitinib. The case of a patient with metastatic

\footnotetext{
* Correspondence: takeshi.yuasa@jfcr.or.jp

'Department of Urology, Cancer Institute Hospital, Japanese Foundation for Cancer Research, Ariake, Tokyo 135-8550, Japan

Full list of author information is available at the end of the article
}

RCC who developed recall pneumonitis on the 14th day of systemic sunitinib treatment is described here. There is currently only one report of recall pneumonitis during sunitinib therapy in the literature [4]. The time of onset in that case was quite different from that in the present case.

\section{Case presentation}

A 65-year-old man with clear cell RCC and lung metastasis was initially treated by right nephrectomy on November, 2011 (pT2b, N0, M1). On January, 2012, during the follow-up period, bilateral lower limb paralysis occurred due to spinal compression from RCC bone metastasis (Figure 1A). He underwent radiation therapy on his thoracic vertebrae (Th5-8) with a total dose of 24 Gy that was divided into six fractions (Figure $1 \mathrm{~B}$ and $\mathrm{C}$ ). Sunitinib (37.5 mg) was started 14 days after completing the radiation therapy. On the 14th day of sunitinib treatment, the patient complained of high fever and the chest CT disclosed bilateral lung consolidation (Figure 2A). At this time, blood culture examinations did not reveal any significant bacteria or fungi, and serum levels of anti-

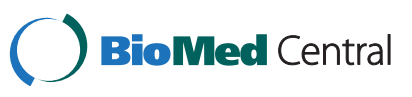




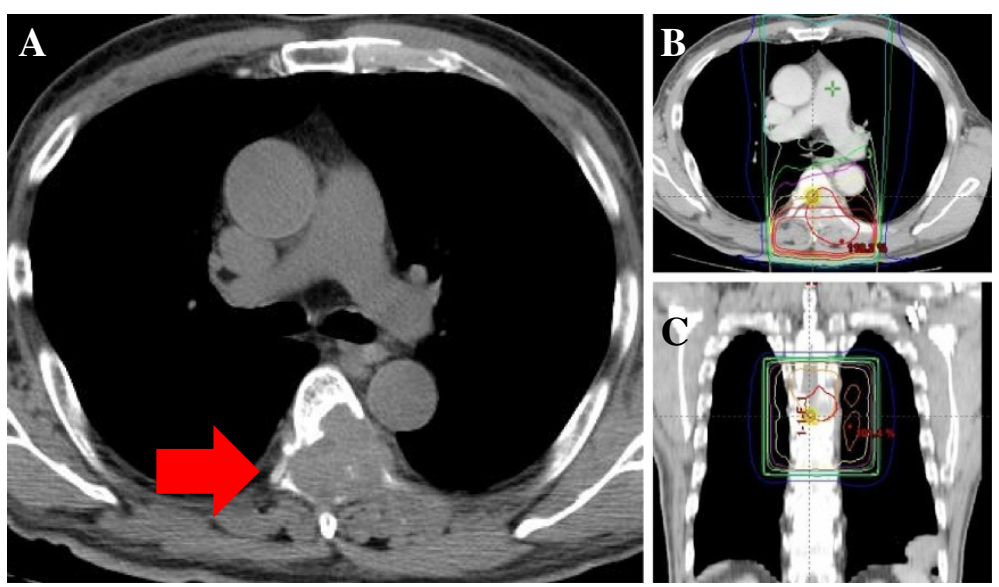

Figure 1 The patient underwent radiation therapy for spinal bone metastasis. The spinal compression from the RCC bone metastasis (A). The irradiated region is shown in the horizontal section (B) and the coronal section (C).

cytomegalovirus antibody, pulmonary surfactant protein-D, and sialylated carbohydrate antigen KL-6 were within the normal range. Although he was observed without sunitinib administration, the patient developed progressive fever with worsening of dyspnea and general weakness (Figure 2B). Treatment with pulse administration of prednisolone 1,000 $\mathrm{mg}$ for 3 days was initiated along with antifungal agent (voriconazole) and antibiotic (meropenem hydrate) treatment. After the steroid pulse therapy, the symptoms gradually improved over 7 days. The radiological findings revealed that the interstitial filtration was localized within the previously irradiated area (Figure 2C). Therefore, the patient was diagnosed with radiation recall pneumonitis that was induced by sunitinib. During the treatment, serial sputum specimen and blood culture examinations did not reveal any significant bacteria or fungus. The patient recovered from the interstitial lung disease completely during the

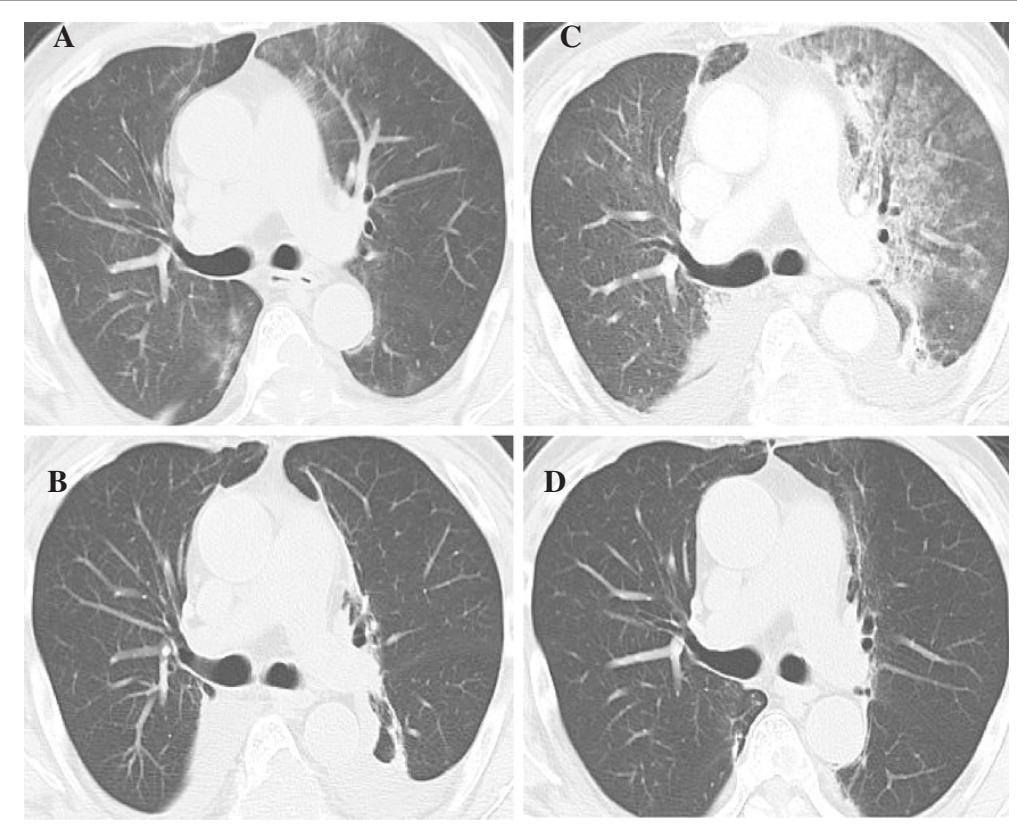

Figure 2 Radiation recall pneumonitis induced by sunitinib. On the 14th day of sunitinib induction, bilateral lung consolidation appeared (A) During follow-up without sunitinib, consolidation developed along with progressive fever (B). After steroid pulse therapy, the CT findings revealed that the interstitial filtration was localized in the previously irradiated area (C). No evidence of relapse of recall pneumonitis during sorafenib treatment was observed (D). 
2 months of follow-up (Figure 2D). Currently, he is undergoing targeted therapy with another tyrosine kinase inhibitor, sorafenib, with no evidence of relapse of the tyrosine kinase inhibitor-associated recall pneumonitis.

\section{Discussion}

The radiation recall phenomenon has been reported in the skin, lung, and mucosa [5-7]. Causal agents have been reported to be cytotoxic agents, including actinomycin D, gemcitabine, taxanes, and anthracyclines, and the estrogen receptor antagonist tamoxifen [5-7]. In most cases, dermatitis is reported. Radiation recall pneumonitis appears to be relatively rare. The mechanism of the recall response remains to be clarified. It has been proposed that it is the result of the inhibition of cellular recovery by cytotoxic agents after damage caused by radiation [6-8]. It is possible that prevention of angiogenesis by sunitinib reduces the recovery from the radiation-induced cell damage. Alternatively, radiation injury may induce hypersensitivity to sunitinib [6-8]. Alternatively, radiation injury may induce hypersensitivity to sunitinib [6-8].

A case of interstitial pneumonitis during targeted therapy with sunitinib is reported here. Pulmonary damage commonly occurs with doses more than $30 \mathrm{~Gy}$ and usually develops 1-6 months after cessation of radiation therapy to the lung. In the present case, the total dose of the radiation that was administered to the patient was only 24 Gy. Since the volume of lung that received more than 20 Gy (V20) was only $2 \%$ (Figure $1 \mathrm{C}$ ) and the patient had received radiation therapy within one month before the onset of the pneumonitis, it was considered to be unlikely to be radiation-induced pneumonitis. Therefore, it was concluded that the pneumonitis was recall pneumonitis induced by sunitinib. There is currently only one report of recall pneumonitis during sunitinib therapy [4]. In that report, a female patient with RCC developed pneumonitis more than 6 months after sunitinib induction and the completion of radiation therapy (30 Gy: 5 fraction) [4]. Her pneumonitis resolved completely when the dose of sunitinib was reduced (from $50 \mathrm{mg} /$ day to $37.5 \mathrm{mg} /$ day); steroids were not administered [4]. Thus, this case differs from the present case in terms of the time of onset, the radiation dose that was administered, and the severity of the pneumonitis.

Although how sunitinib interacts with radiation therapy remains unclear, this interaction may lead to synergistic toxicities. There has been a report of lethal small bowel perforation after radiation for lumbar metastasis during sorafenib administration; moreover, the case of a lethal bronchial fistula that occurred after radiation for mediastinum lymph node metastasis during sunitinib therapy was published $[9,10]$. The current knowledge about the combination of tyrosine kinase inhibitors with radiation therapy has been reviewed by Niyazi et al. [7]. At present, it seems that none of the tyrosine kinase inhibitors have been approved for simultaneous use with radiation therapy.

\section{Conclusions}

In conclusion, a case of early onset recall pneumonitis during targeted therapy with sunitinib was reported here. At present, how sunitinib interacts with radiation therapy remains unclear. The possibility that tyrosine kinase inhibitor therapy, including with sunitinib, after radiation therapy may lead to adverse effects should be kept in mind.

\section{Consent}

Written informed consent was obtained from the patient for publication of this Case report and any accompanying images. A copy of the written consent is available for review by the Series Editor of this journal.

\section{Abbreviations}

RCC: Renal cell cancer; VEGFR: Vascular endothelial growth factor receptor; PDGFR: Platelet derived growth factor receptor.

\section{Competing interests}

The authors declare that they have no competing interests.

\section{Authors' contributions}

Conception and design: TY; Manuscript writing: TY; Final approval: SK, GS, SY, KK, KM, TK, SH, and JY; Pathological explorations: Nl; Patient's management: TY, SK, GS, SY, JY; All authors read and approved the final manuscript.

\section{Acknowledgments}

The work was partly supported by the Smoking Research Foundation, the Takeda Science Foundation, and Grants-in-Aid for Scientific Research from the Ministry of Education, Culture, Sports, Science and Technology, Japan.

\section{Author details}

${ }^{1}$ Department of Urology, Cancer Institute Hospital, Japanese Foundation for Cancer Research, Ariake, Tokyo 135-8550, Japan. ${ }^{2}$ Department of Thoracic Medical Oncology, Cancer Institute Hospital, Japanese Foundation for Cancer Research, Ariake, Tokyo 135-8550, Japan. ${ }^{3}$ Department of Radiation Oncology, Cancer Institute Hospital, Japanese Foundation for Cancer Research, Ariake, Tokyo 135-8550, Japan. ${ }^{4}$ Department of Infectious Diseases, Cancer Institute Hospital, Japanese Foundation for Cancer Research, Ariake, Tokyo 135-8550, Japan.

Received: 25 September 2012 Accepted: 27 December 2012

Published: 2 January 2013

\section{References}

1. Srinivasan $R$, Linehan WM: Treatment of advanced renal cell carcinoma. In Campbell-Walsh Urology. 10th edition. Edited by Kavoussi LR, Novick AC, Partin AW, Peters CA, Wein AJ. New York: Saunders; 2011:1475-1491.

2. Yuasa T, Takahashi S, Hatake K, Yonese J, Fukui I: Biomarkers to predict response to sunitinib therapy and prognosis in metastatic renal cell cancer. Cancer Sci 2011, 102:1949-1957.

3. Yuasa T, Tsuchiya N, Horikawa Y, Narita S, Inoue T, Urakami S, Yamamoto S, Yonese J, Takahashi S, Hatake K, Fukui I, Habuchi T: Clinical efficacy and prognostic factors for overall survival in japanese patients with metastatic renal cell cancer treated with sunitinib. BJU Int 2011, 109:1349-1354.

4. Seidel C, Janssen S, Karstens JH, Welte T, Morgan M, Ganser A, Grünwald V: Recall pneumonitis during systemic treatment with sunitinib. Ann Oncol 2010, 21:2119-2120.

5. Parry BR: Radiation recall induced by tamoxifen. Lancet 1992, 340:49.

6. Burris HA 3rd, Hurtig J: Radiation recall with anticancer agents. Oncologist 2010, 15:1227-1237. 
7. Niyazi M, Maihoefer C, Krause M, Rödel C, Budach W, Belka C: Radiotherapy and "new" drugs-new side effects? Radiat Oncol 2011, 21:177.

8. McDonald S, Rubin P, Phillips TL, Marks LB: Injury to the lung from cancer therapy: clinical syndromes, measurable endpoints, and potential scoring systems. Int J Radiat Oncol Biol Phys 1995, 31:1187-1203.

9. Peters NJB, Richel DJ, Verhoeff JJC, Stalpers L: Bowel perforation after radiotherapy in a patient receiving sorafenib. J Clin Oncol 2008, 26:2405-2406.

10. Basille D, Andrejak M, Bentayeb $H$, Kanaan M, Fournier C, Lecuyer E, Boutemy M, Garidi R, Douadi Y, Dayen C: Bronchial fistula associated with sunitinib in a patient previously treated with radiation therapy. Ann Pharmacother 2010, 44:383-386.

doi:10.1186/1471-2407-13-3

Cite this article as: Yuasa et al.: Early onset recall pneumonitis during targeted therapy with sunitinib. BMC Cancer 2013 13:3.

\section{Submit your next manuscript to BioMed Central and take full advantage of:}

- Convenient online submission

- Thorough peer review

- No space constraints or color figure charges

- Immediate publication on acceptance

- Inclusion in PubMed, CAS, Scopus and Google Scholar

- Research which is freely available for redistribution 